\title{
Embedding partial Steiner triple systems so that their automorphisms extend
}

\author{
Peter J. Cameron \\ School of Mathematical Sciences \\ Queen Mary, University of London \\ Mile End Road \\ London E1 4NS \\ U.K.
}

\begin{abstract}
It is shown that there is a function $g$ on the natural numbers such that a partial Steiner triple system $U$ on $u$ points can be embedded in a Steiner triple system $V$ on $v$ points, in such a way that all automorphisms of $U$ can be extended to $V$, for every admissible $v$ satisfying $v>g(u)$. We find exponential upper and lower bounds for $g$.
\end{abstract}

\section{Introduction}

A partial Steiner triple system on a set $U$ of $u$ points is a collection of 3-subsets (called triples) of $U$ such that any 2-subset of $U$ is contained in at most one triple. If we replace "at most" by "exactly" in this definition, we have a Steiner triple system. The number $u$ is the order of the system. As is well known, Kirkman proved that a Steiner triple system of order $u$ exists if and only if $u$ is congruent to 1 or 3 mod 6. (Such numbers are called admissible.)

We abbreviate "partial Steiner triple system" and "Steiner triple system" to PSTS and STS respectively.

An automorphism of a PSTS on a set $U$ is a permutation of $U$ which maps triples to triples.

Suppose we are given PSTS on sets $U$ and $V$. We say the first system is embedded in the second if $U \subseteq V$ and the triples of the first system are precisely the triples of the second system which are contained in $U$. (Such an embedding is sometimes called 'faithful', but I do not consider any other kind of embedding.) 
The embedding is smooth if every automorphism of the first system can be extended to the second.

Embeddability of partial STS in STS is a well-understood topic. Doyen and Wilson [3] showed that a STS of order $u$ can be embedded in an STS of any admissible order greater than $2 u$. Lindner [4] conjectured that, more generally, a PSTS of order $u$ can be embedded in an STS of any admissible order greater than $2 u$. The conjecture has recently been proved by Bryant [2], though this is not yet published.

Automorphisms of Steiner triple systems have also received much attention. For example, Mendelssohn [5] showed that every finite group is the automorphism group of a finite Steiner triple system, and Babai [1] showed that almost all Steiner triple systems have trivial automorphism group.

What about the analogue of Lindner's conjecture for smooth embeddings? In particular, we could ask whether the following is true:

(a) There is a function $f$ such that any PSTS of order $u$ can be smoothly embedded in some STS of order $f(u)$.

(b) There is a function $g$ such that any PSTS of order $u$ can be smoothly embedded in some STS of any admissible order $v>g(u)$.

Below, I prove these two statements. For the first, a best possible result is obtained:

Theorem 1 For $u \geq 4$, a partial Steiner triple system of order $u$ is smoothly embeddable in a Steiner triple system of order $2^{u-1}-1$. There is a PSTS of order $u$ which is not smoothly embeddable in any smaller STS.

Given this result, to establish the second statement it suffices to show that there is a function $h$ such that a STS of order $v$ can be smoothly embedded in an STS of any admissible order at least $h(v)$. This is accomplished by the next result:

Theorem 2 Let $V$ be a Steiner triple system of order $v$. Then for any admissible $w>6 v^{2}$, there is a Steiner triple system of order $w$ in which $V$ can be smoothly embedded.

Thus the second statement above holds, with $g(u) \leq 6\left(2^{u-1}-1\right)^{2}$. By Theorem $1, g(u) \geq 2^{u-1}-3$. So $g$ is bounded above and below by exponential functions.

The proofs of the theorems actually give a stronger result than stated. Not only do all automorphisms of the subsystem extend, but there is a group of automorphisms of the whole system which faithfully induces the automorphism group 
of the subsystem. However, it is not the case that the automorphism groups of the system and subsystem are equal in general. (In the construction used to prove Theorem 1, if the subsystem contains no triples, its automorphism group is the symmetric group $S_{u}$, while that of the embedding system is the general linear group GL $(u-1,2)$.)

The bound in Theorem 2 is not best possible. It shows, for example, that the Fano plane (the unique STS of order 7) is smoothly embeddable in a STS of any admissible order greater than 294; it is not difficult to reduce this to 194 . I do not know what the best possible value is.

\section{Proof of Theorem 1}

We begin by showing that the function $2^{u-1}-1$ cannot be improved. This relies on two simple facts:

(a) the fixed points of an automorphism of a STS carry a STS;

(b) if a STS of order $v$ has a proper subsystem of order $w$, then $v \geq 2 w+1$.

Now take the trivial PSTS on $u$ points with no triples; its automorphism group is the symmetric group $S_{u}$. Suppose that we have a smooth embedding in a STS of order $v$. For $i=1, \ldots, u-1$, let $G_{i}$ be the stabiliser of $1, \ldots, i$. Let $v_{i}$ be the number of fixed points of $G_{i}$. Then $v_{i}>v_{i-1}$, so $v_{i} \geq 2 v_{i-1}+1$. Since $v_{1} \geq 1$, a simple induction gives $v_{i} \geq 2^{i}-1$, so that

$$
v \geq v_{u-1} \geq 2^{u-1}-1
$$

Now we turn to the construction. By inspection, both PSTS of order 4 are smoothly embeddable in the Fano plane, so we may assume that $u \geq 5$. Let $U$ be the point set of the PSTS.

We give first an embedding in a system of order $2^{u}-1$. Start with the projective geometry, whose points are all the non-empty subsets of $U$, the triples being all $\{A, B, C\}$ whose symmetric difference is empty. Any permutation of $U$ extends uniquely to an automorphism of this system.

For each triple $\{a, b, c\}$ of the PSTS on $U$, we replace the triples $\{a, b, a b\}$, $\{b, c, b c\},\{a, c, a c\}$ and $\{a b, a c, b c\}$ by $\{a, b, c\},\{a, a b, a c\},\{b, a b, b c\}$ and $\{c, a c, b c\}$. (By abuse of notation we write $a$ and $a b$ for $\{a\}$ and $\{a, b\}$ respectively.) The new triples cover the same pairs as the old ones, so we again have a STS. Clearly the given PSTS is embedded in it. Moreover, given an automorphism of the PSTS, its unique extension to an automorphism of the projective geometry is also an automorphism of the new system. (We only have to check the altered triples. For 
example, if $\{a, a b, a c\}$ is a new triple and $g$ an automorphism mapping $a$ to $a_{1}, b$ to $b_{1}$, and $c$ to $c_{1}$, then $\{a, b, c\}$ belongs to the PSTS, and hence so does $\left\{a_{1}, b_{1}, c_{1}\right\}$; thus $\left\{a_{1}, a_{1} b_{1}, a_{1} c_{1}\right\}$ is also a new triple.)

We can modify this construction to reduce the dimension of the projective geometry by 1: take the points to be partitions $\left\{A, A^{\prime}\right\}$ of $U$ into two non-empty parts, where three such partitions $\left\{A, A^{\prime}\right\},\left\{B, B^{\prime}\right\}$ and $\left\{C, C^{\prime}\right\}$ form a triple whenever the symmetric difference of $A, B, C$ is $\emptyset$ or $U$. )This STS has order $2^{u-1}-1$.) Since $u \geq 5$, such a partition has at most one part of size 1 or 2 , so the switches we make don't interfere with one another.

\section{Proof of Theorem 2}

There are many standard constructions of STS from smaller ones. We must use only constructions which provide smooth embeddings. We show the following.

Lemma 3 Let $V$ be a STS of order $v$, and let $x$ and $y$ be admissible positive integers with $y \geq 2 x+1$. Then there is a STS of order $x+v(y-x)$ in which $V$ is smoothly embedded.

Proof By the theorem of Doyen and Wilson [3], there is a STS on a set $Y$ of order $y$ containing a subsystem on a subset $X$ of order $x$.

Take $S=X \cup(V \times(Y \backslash X))$. Let $L$ be a totally symmetric Latin square of order $y-x$ satisfying the condition that the entry in the first row and first column is 1 . (A Latin square is totally symmetric if, whenever the $(i, j)$ entry is $k$, the same is true after any permutation of $\{i, j, k\}$. For example, let $A=\left\{a_{1}, \ldots, a_{y-x}\right\}$ be an abelian group of order $y-x$, with identity $a_{1}$; let $L$ have $(i, j)$ entry $k$ if $a_{i} a_{j} a_{k}=a_{1}$.) Number the elements of $Y \backslash X$ as $q_{1}, \ldots, q_{y-x}$. Now take triples on $S$ of the following two types:

(a) the images of the triples of $Y$ under the bijection to $X \cup(\{p\} \times(Y \backslash X))$ given by $r \mapsto r$ for $r \in X, q \mapsto(p, q)$ for $q \in Y \backslash X$, for each $p \in V$;

(b) those of the form $\left\{\left(p_{1}, q_{i_{1}}\right),\left(p_{2}, q_{i_{2}}\right),\left(p_{3}, q_{i_{3}}\right)\right\}$ whenever $\left\{p_{1}, p_{2}, p_{3}\right\}$ is a triple of $V$ and the $\left(i_{1}, i_{2}\right)$ entry of $L$ is $i_{3}$.

This is easily seen to be an STS, and the set $\left\{\left(p, q_{1}\right): p \in V\right\}$ carries a subsystem isomorphic to the given subsystem on $V$. Furthermore, if $g$ is an automorphism of $V$, then we extend it to $S$ by defining

$$
\begin{aligned}
r^{g}=r & \text { for } \quad r \in X, \\
(p, q)^{g}=\left(p^{g}, q\right) & \text { for } \quad p \in V, q \in Y \backslash X .
\end{aligned}
$$


It is readily checked that this is an automorphism of $S$, acting on the subsystem $\left\{\left(p, q_{1}\right): p \in V\right\}$ in the same way as it acts on $V$.

Now we apply this construction to realise any admissible order greater than $6 v^{2}$. First, consider orders congruent to $1 \bmod 6$. Take $x$ and $y$ both congruent to 1 mod 6. Consider a fixed value of $y-x$, say $y-x=6 a$. By choosing $x=$ $1, \ldots, 6 a-5$, we realise the orders

$$
1+6 a v, 7+6 a v, \ldots, 6 a-5+6 a v .
$$

For $y-x=6(a+1)$, we realise

$$
1+6(a+1) v, 7+6(a+1) v, \ldots, 6 a+1+6(a+1) v .
$$

In order not to leave any gaps, we require that these intervals abut or overlap. This occurs as long as

$$
6 a+1+6 a v \geq 1+6(a+1) v,
$$

that is, $a \geq v$. So we can achieve all orders congruent to $1 \bmod 6$ from $6 v^{2}+1$ onwards.

Exactly the same argument deals with orders congruent to 3 mod 6: we obtain all such orders from $6 v^{2}+3$ onwards.

So the theorem is proved.

\section{References}

[1] L. Babai, Almost all Steiner triple systems are asymmetric, in Topics in Steiner systems (ed. C. C. Lindner and A. Rosa), Ann. Discrete Math. 7, Elsevier, Amsterdam, 1979, pp. 37-39.

[2] D. Bryant, talk at 19th ACCMCC, Taupo, New Zealand, December 2004.

[3] J. Doyen and R. M. Wilson, Embeddings of Steiner triple systems, Discrete Math. 5 (1973), 229-239.

[4] C. C. Lindner, A partial Steiner triple system of order $n$ can be embedded in a Steiner triple system of order $6 n+3, J$. Combinatorial Theory Ser. A 18 (1975), 349-351.

[5] E. Mendelsohn, On the groups of automorphisms of Steiner triple and quadruple systems, J. Combinatorial Theory Ser. A 25 (1978), 97-104. 